\title{
mGluR5-dependent acetylcholine, serotonin, and norepinephrine mediated modulation of synaptic eligibility traces into LTD in mice visual cortex
}

Shumsuzzaman Khan ( $\sim$ p53p35dna@yahoo.com )

Johns Hopkins University

\section{Research Article}

Keywords: LTD, Acetylcholine, Serotonin, Norepinephrine, Visual cortex, MPEP

Posted Date: January 15th, 2021

DOl: https://doi.org/10.21203/rs.3.rs-147167/v1

License: (c) (1) This work is licensed under a Creative Commons Attribution 4.0 International License.

Read Full License 


\section{Abstract}

In reward-based learning, synaptic eligibility traces are a well-defined theoretical solution for the conversion of initial co-activation of pre and postsynaptic neurons into long-term changes in synaptic strength by reward-linked neuromodulators. However, the types of neuromodulators involved in such a phenomenon in mouse visual cortex remain unknown. To characterize the Ex vivo condition, we used optogenetic stimulation of channelrhodopsin-(ChR2) expressing Cre/Ai32(ChR2-eYFP); Tph2Cre/Ai32(ChR2-eYFP); Thi-Cre/Ai32(ChR2-eYFP) homozygous mice, which release acetylcholine, serotonin, and norepinephrine, respectively. With these mice it is possible to measure the transformation of eligibility traces into long-term changes by endogenous neuromodulators. Here we delineated that layer 2/3 neurons in the visual cortex showed no LTD after conditioning with paired-pulse low-frequency stimulation (ppLFS; $2 \mathrm{~Hz}, 15 \mathrm{~min}$ ). However, if conditioning was paired with acetylcholine, serotonin, or norepinephrine release upon $473 \mathrm{~nm}$ optical stimulation in brain slices, LTD occurs in every case. Thus, our data suggests a new pathway to connect the gap between stimulus and reward. Moreover, we found that stimulation by theta-glass or metal stimulators evoked IPSC traces with the same amplitudes but differences in decay kinetics, further questioning the appropriate use of stimulators in brain slices for evoking an event.

\section{Introduction}

To avoid punishment and to maximize reward, memories in neuronal networks are thought to be exiguously encoded. Different types of memories are encoded in different brain regions, however, only a small number of neurons are required to encode a distinct memory $[1,2]$. Interestingly, primary visual cortical neurons can illustrate eward timing in response to sensory cues through a course of conditioning [3]. Although it is poorly understood how cortical neurons represent reward timing activity, it is thought that reinforcement learning plays a crucial role, where the neural activity of reinforcement signal links with the behavioral outcome [4]. Previously, it has been reported that a combination of presynaptic trains of high-frequency cortical stimulation with striatal postsynaptic depolarization resulted in long-term depression (LTD) $[5,6]$.

Synaptic eligibility traces are induced by silent or transient synaptic activity which is transformed into long term synaptic strength at a later stage by the action of different neuromodulators. Neuromodulators, such as acetylcholine (ACh), norepinephrine (NE) and serotonin (SE) are good candidates for reinforcement because of their capacity to transduce signaling of behavioral output through the visual cortex [7,8]. Acetylcholine is associated with learning and memory [9], is highly expressed in the visual cortex, and ultimately modulates the plasticity of the visual cortex [10]. Moreover, ACh is responsible for the organization of the circuit map via tuning of neural properties in the cortex $[11,12]$. In the hippocampus, ACh can induce both LTP and LTD depending on the induction protocol and concentration of ACh $[13,14,15]$. Additionally, reduced hippocampal acetylcholine levels are associated with impaired fear learning and memory [16]. Thus, ACh plays a crucial role in the control of brain states, attention, and learning. lonotropic nicotinic receptors and metabotropic muscarinic receptors are the main mediators of 
ACh function in the cortex. In an orientation discrimination task, optogenetic stimulation of cholinergic neurons in the visual cortex (V1) enhances visual responsiveness and performance [17]. Other behavioral factors, such as unexpected rewards, punishment, and arousal levels, are also influenced by cholinergic neurons [18]. Pharmacological blockers of cholinergic signaling impair recognition memory and spatial memory [19].

Serotonin (SE) is a neuromodulator that is highly associated with the cognitive, affective, and behavioral functions of the brain. It has been reported that an animal's patience for future rewards is associated with enhanced serotonergic activity in the dorsal raphe nucleus (DRN) $[20,21,22]$. Failure to associate a given reward or punishment with past events (action choices) represents impairment in the serotonergic system. Slower associative learning reflects lower serotonergic condition, when actions were followed by a delayed punishment. Thus, SE regulates the association of time with aversive outcomes (punishment) to past events [23]. Midbrain serotonin neurons are affected by cocaine, heroin, nicotine, and MDMA. MDMA and cocaine cause long-lasting inhibition of serotonin neurons [24]. Hippocampal dentate gyrus (DG) granule cells (GCs) exhibit an antidepressant response in the presence of serotonin [25]. Moreover, optogenetic stimulation of hippocampal CA1 serotonergic terminals enhances spatial memory via increased excitatory transmission at CA3-to-CA1 synapses [26]. Interestingly during sustained goaldirected behavior, suppression of ventral hippocampus revealed increased activity in the median but not the dorsal raphe, suggesting that serotonergic signaling is the underlying mechanism of ventral hippocampus suppression during that behavior. In reward-based learning, serotonergic signaling modulates spike time dependent (STD)-long-term depression (LTD) at thalamostriatal synapses by downregulating the activity of the 5-HT4 receptor subtype.

Norepinephrine is associated with the control of rewarding stimuli and arousal $[27,28]$. In the case of emotional learning, NE enhances the phosphorylation of GluR1 to facilitate the translocation of AMPAR, thus lowering the threshold for LTP induction. Moreover, GluR1 phosphomutant and wild type (C57BL/6) mice showed similar phosphorylation patterns in the non-stressful (neutral) condition, suggesting that learning at the basal condition may not involve NE-mediated plasticity [29]. In anesthetized mice, increased microglial surveillance is associated with reduced norepinephrine signaling, while at basal physiological conditions in awake mice microglial surveillance is suppressed by norepinephrine [30]. In a retrograde neuronal-glial circuit, norepinephrine activates $\mathrm{CRH}$ neurons to enhance the calcium response in astrocytes, which causes release of ATP and activation of GABAergic and glutamatergic neurons [31].On the other hand, increasing dendritic excitability via activation of adrenergic signalling facilitates associative learning [32]. At excitatory synapses $\beta$-adrenergic signalling regulates spike-timing-dependent (STD) LTP expression via insertion of AMPAR's [29].

Two distinct forms of long-term depression (LTD) have been reported in the hippocampus: the NMDA receptor dependent LTD and metabotropic glutamate receptor dependent (mGluRs) LTD [33]. Moreover, in hippocampal neurons, mitochondrial activation of caspase-3 is required for AMPA receptor internalization and, thus, for LTD [34]. LTD, a de novo decrease in synaptic efficacy, is considered the cellular mechanism underlying the extinction of fear memory $[35,36]$. Besides fear memory, hippocampal LTD is also 
associated with impairment of working memory by cannabinoids [37]. In the ventral tegmental area (VTA), dopamine blocks LTD via activation of cyclic AMP-dependent protein kinase (PKA) [38]. In the cerebellum, LTD depresses the burst of presynaptic action potentials in a presynaptic NMDAR manner [39]. On the other hand, on the postsynaptic side, the expression of cerebellar LTD requires clathrinmediated internalization of AMPARs [40]. Cerebellar LTD also requires enhanced cellular concentrations of $\mathrm{Ca}^{2+}$ [41]. Moreover, optogenetic stimulation of Purkinje cells in cerebellum enhanced nitric oxide (NO), mimicking activation of the parallel fibers (PF) with a brief postsynaptic depolarization to induce LTD. NMDAR dependent LTD is also seen in the thalamocortical synapses in the barrel cortex [42]. Thus, LTD is well studied in the hippocampus and other regions of the brain, however, there is a lack of understanding about the cholinergic, serotonergic, and noradrenergic modulation of LTD in the visual cortex.

Several experiments have been carried out to map the spread of the electrical stimulation from the tip of microelectrode to discrete regions of the central nervous system (CNS). A monopolar electrode, a bipolar electrode, and a concentric configuration were used in different experiments. The monopolar electrode showed more reduction in the spread of electrical stimulation compared with the bipolar electrode. The extent of the electrical stimulation spread was dependent on various experimental conditions including the diameter of the microelectrode tip [43]. However, the concentric bipolar electrode showed a reduction in the extent of stimulus spread. The evoked discharge in neural circuit analysis is induced by electrical stimulation either via a metal electrode or by a theta glass electrode (double-barreled glass pipettes). It has been reported that double-barreled ion-selective microelectrodes $(\mathrm{Na} / \mathrm{K})$ and concentric ion-selective microelectrodes $(\mathrm{Na} / \mathrm{K})$ show similar response in amplitude but concentric sodium microelectrodes show greater response time with higher signal-to-noise compared to the double-barreled design [44]. Still, there a lack of understanding about evoked IPSCs in the hippocampal dentate gyrus (DG) via bipolar tungsten electrodes or borosilicate theta glass electrodes. Here I showed that IPSCs evoked by the metal electrode or by a theta glass electrode showed similar amplitude with different decay kinetics.

\section{Materials And Methods}

\subsection{Animal and tissue}

\subsubsection{Transgenic mice}

ChAT-IRES-Cre mice on C57BL/ 6 genomic background express Cre recombinase specifically in cholinergic neurons without altering the endogenous acetylcholine release. On the other hand, Ai32 mice express an improved (due to an H134R mutation in channelrhodopsin: ChR2) ChR2/eYFP fusion protein. To examine the Cre-dependent expression of the ChR2, we bred ChAT-IRES-Cre mice with Ai32 (ChR2-eYFP) reporter mice to generate ChAT-Cre/Ai32(ChR2-eYFP) mice. Homozygous mice were selected for optogenetic experiments, while wild-type (C57BL/6) mice were used as control. In a similar approach, Tph2Cre/Ai32(ChR2-eYFP) mice and Thi-Cre/Ai32(ChR2-eYFP) mice were generated. Both sexes were used in experiments. All experiments and procedures were performed following protocols approved by the Johns Hopkins University Animal Care and Use Committee. All subjects were fed ad libitum. 


\subsubsection{Slice preparation}

Wild-type C57BL/6J mice and transgenic Chat-cre-ChR2, Tph2-cre-ChR2 and Thi-cre-ChR2 mice on $\mathrm{C} 57 \mathrm{BL} / 6 \mathrm{~J}$ genomic backgrounds, aged P22-40 were anesthetized with isofluorane inhalation before decapitation. The brain was then extracted and glued on the platform of a semiautomatic vibrating blade microtome (VT1200; Leica). The platform was placed in the slicing chamber containing artificial cerebrospinal fluid (ACSF) at $4{ }^{\circ} \mathrm{C}$. Hippocampal slices $(300 \mu \mathrm{m})$ were prepared in ice-cold ACSF, which consisted of (in mM) $125 \mathrm{NaCl}, 3 \mathrm{KCl}, 1.25 \mathrm{NaH}_{2} \mathrm{PO}_{4}, 2 \mathrm{MgSO}_{4}, 2 \mathrm{CaCl}_{2}, 25 \mathrm{NaHCO}_{3}, 10$ glucose, and 0.4 $\mathrm{L}$-Ascorbic acids. We also use ice-cold dissection solution if postnatal day $>30$. Dissection solution composition (in $\mathrm{Mm}$ ): 212.7 sucrose, $3 \mathrm{KCl}, 1.25 \mathrm{NaH}_{2} \mathrm{PO}_{4}, 3 \mathrm{MgCl}_{2}, 1 \mathrm{CaCl}_{2}, 26 \mathrm{NaHCO}_{3}$, and 10 dextrose. All slides were incubated in a custom-made interface holding a chamber submerged in ACSF at $30^{\circ} \mathrm{C}$ for 30 minutes and then maintained at room temperature up to $8 \mathrm{~h}$ until they were transferred to the recording chamber. Slices were superfused $(2 \mathrm{ml} / \mathrm{min})$ with ACSF at $29-31^{\circ} \mathrm{C}$. All solutions were saturated with $95 \% \mathrm{O}_{2}, 5 \% \mathrm{CO}_{2}$.

\subsection{Electrophysiology}

\subsubsection{LTD and evoked inhibitory postsynaptic current recordings}

Slices were visualized through an infrared-sensitive CCD camera with a $40 \times$ water-immersion lens (Olympus) and recorded using whole-cell techniques (MultiClamp 700B Amplifier). Slices were allowed 5$10 \mathrm{~min}$ to equilibrate before recording. Recording electrodes were pulled on a horizontal puller (Sutter P97). Evoked EPSPs (eEPSPs) were recorded from layer $2 / 3$ pyramidal cells by whole-cell current-clamp method, while elPSC were recorded from DG granule cells of the hippocampus by whole-cell voltageclamp method. A Borosilicate Theta glass electrode or a concentric bipolar stimulating electrode with a tip diameter of $125 \mu \mathrm{m}$ was placed in the hilus to evoke inhibitory responses onto DG granule cells of the hippocampus. The same metal electrode was placed in layer 4 to evoke excitatory responses onto pyramidal cells in layer $2 / 3$. The distance between the recording- and stimulating-electrode was kept at 80-120 $\mu \mathrm{m}$. Patch pipettes (3-4 M $\Omega$ ) were filled with the internal solution consisting of the following (in $\mathrm{mM}$ ): $130 \mathrm{CsMeSO}, 20 \mathrm{CsCl}, 10$ HEPES buffer, 4 Mg-ATP, $0.3 \mathrm{Na}$-GTP, 10 disodium phosphocreatine, and $0.2 \mathrm{EGTA}, \mathrm{pH} 7.3$ with $\mathrm{CsOH}$, and $288 \mathrm{mOsm}$. Data were digitized at $10 \mathrm{kHz}$ and filtered at $3 \mathrm{kHz}$ by using Igor Pro (WaveMetrics). Pairing protocol used to induce LTD: blue light $(473 \mathrm{~nm})$ stimulation $(1 \mathrm{~Hz}, 15$ $\mathrm{min}$ ) paired with paired-pulse low-frequency stimulation (ppLFS, $2 \mathrm{~Hz}, 15 \mathrm{~min}$ ). Baseline amplitude for $10-$ 15 min before and for 30-40 min following paired stimulation, was monitored by a test pulse at $0.05 \mathrm{~Hz}$. A hyperpolarizing step of $-10 \mathrm{mV}$ every $60 \mathrm{~s}$ was used to monitor access resistance in whole-cell recordings. Neurons were excluded from the experiment if the access resistance changed more than $10 \%$ or input resistance changed more than 15\%. Evoked EPSPs were recorded in the presence of NMDAR blocker AP5 $(25 \mu \mathrm{M})$, while the elPSCs were recorded in the presence of DNQX $(10 \mu \mathrm{M})$ and AP5 $(25 \mu \mathrm{M})$.

\subsubsection{Stimulating electrodes}


A concentric bipolar tungsten stimulation electrode (World Precision Instruments, Sarasota, FL) or Clark Borosilicate Theta glass electrode (Warner Instruments) was used to evoke IPSC in the experiments. The theta glass electrode was processed with a pipette puller (P-97 micropipette puller, Sutter Instrument) to produce tapered tips. To evoke the IPSC, the theta micropipette was used as a bipolar microelectrode to pass the electrical stimulation. The theta glass electrode was injected with ACSF solution for conduction and connected to an amplification system.

\subsection{Drugs and Reagents}

All drugs and buffers for intracellular and extracellular solutions, as well as ATP, GTP, phosphocreatine, MPEP, AP5, DNQX, and picrotoxin, were from Sigma.

\subsection{Statistical Analysis}

All data are shown as mean \pm SEM. Statistical significance was assessed by using unpaired t-tests in Origin Pro/GraphPad Prism. $P<0.05$ was considered to be statistically significant $\left(* P<0.05\right.$, ${ }^{*} * \mathrm{P}<$ 0.001). Data for which a specific P-value is not indicated are not significantly different. The EPSP amplitude was normalized to the mean baseline amplitude to measure LTD. Mean normalized EPSP amplitude of 25-30 min after paired stimulation was used to define LTD magnitudes.

\section{Results}

\subsection{Paired-pulse low frequency stimulation (ppLFS) in the presence of a NMDAR antagonist is not sufficient to induce LTD in mouse or rat visual cortex}

Low-frequency stimulation (LFS) is used to induced LTD in brain slices depending on the experimental protocol and the age of the animal. Usually, LTD is dependent on the pattern of conditioning and/or frequency of stimulation and activation of NMDA receptors [45]. LTD of the visual cortex represents reduced responses to the paired stimulus. Thus the temporal variation of pre-and postsynaptic activity plays a crucial role in cortical modulation [46]. In our experiment, whole-cell recordings were made from 6 neurons in separate brain slices from 10 mice. Wild type (C57BL/6) mice from P22 to P40 showed no LTD in layer $2 / 3$ visual cortex after pairing blue light $(473 \mathrm{~nm})$ stimulation with paired-pulse lowfrequency stimulation (ppLFS) $(2 \mathrm{~Hz}, 15 \mathrm{~min})(97.5 \pm 1.9 \%$ baseline, $\mathrm{p}=0.42, \mathrm{n}=8$, unpaired t-test) in the presence of the NMDAR antagonist AP5 $(25 \mu \mathrm{M})$ (Fig. 1a). ppLFS with optogenetic stimulation was sufficient to depress the synapses; after the first 15 minutes the average amplitude was $72.3 \pm 2.6 \%$ of baseline. 15 minutes later, the amplitudes of eEPSPs was back to baseline. The average of the last 5 minutes of eEPSPs was $97.5 \pm 1.9 \%$ of baseline. There is no significant difference in the average eEPSP between the baseline and the last 5 minutes after ppLFS with the light stimulation. Thus, no LTD is observed in mouse visual cortex in the presence of an NMDAR antagonist (Fig. 1b).

LTD induction may vary based on the age of the animal, the critical period, and from species to species. Thus, next we asked whether the same induction protocol also reveals the same results in rats as we had 
seen in mice. Here we used rats from the ages of P30-P60. The baseline was recorded for 10 minutes. Then layer 2/3 visual cortex in the rat was stimulated with paired-pulse low-frequency stimulation (ppLFS, 1Hz, $15 \mathrm{~min}$ ) in combination with $473 \mathrm{~nm}$ blue light stimulation. Interestingly, the protocol also failed to induce any LTD in rat visual cortex in the presence of the NMDAR antagonist AP5 (25 $\mu$ M) (Fig. 1c). After 15 minutes, ppLFS with optogenetic stimulation was not sufficient to depress the synapses, the same result seen in the mouse visual cortex. After 20 minutes of stimulation in the rat, we observed a minor decrease of the eEPSPs amplitude from the baseline. The average of the last 5 minutes eEPSPs was $93.4 \pm 1.9 \%$ of baseline. However, there was no significant difference in the average of eEPSPs between the baseline and the last 5 minutes of ppLFS with the light stimulation ( $n=6$, unpaired t-test). Thus, the same induction protocol also failed to induce LTD in the rat virtual cortex (Fig. 1d). Interestingly, when we compare the LTD magnitude (last 5 minutes eEPSP amplitudes) between the mice and rats, we observed a significant reduction in the rat $(93.4 \pm 1.9 \%)$ compared to mouse $(97.5 \pm 1.9$; Fig. 1e). This difference may be due to the age difference between mice (P22 to P40) and rat (P30-P60). Taken together, we conclude that ppLFS is not sufficient to induce LTD during the post-neonatal period in both mice and rat in the presence of an NMDARs antagonist, where the age of the animal may be an issue to determine the threshold of LTD.

\section{2 mGluR5 dependent acetylcholine mediated LTD in mouse visual cortex}

In cholinergic system, the ChAT-Cre/Ai32(ChR2-eYFP) homozygous brain slices were used for the release of acetylcholine, while wild-type (C57BL/6) mice were used as a control. Both ChAT-Cre/Ai32(ChR2-eYFP) homozygous and wild-type (C57BL/6) brain slices went through optical stimulation with ppLFS, and neurons were patched from the layer $2 / 3$ visual cortex. After 10 minutes' baseline recoding, low-frequency stimulation (ppLFS, $1 \mathrm{~Hz}, 15 \mathrm{~min}$ ) in combination with $473 \mathrm{~nm}$ blue light stimulation was applied to the Chat-cre-ChR2 homozygous brain slice in the presence of the NMDAR antagonist AP5. In the presence of acetylcholine, ppLFS induced LTD $(51.5 \pm 5.7 \%$ of baseline; $n=10 ; * \star \star P<0.001$, unpaired t-test) in the visual cortex compare to the baseline value $96.6 \pm 5.5(n=10)$ (Fig. 2a, b). These data suggest that ppLFS is sufficient to induce LTD only in the presence of acetylcholine in mouse visual cortex during the postneonatal period when NMDARs are closed.

mGluR52-Methyl-6-(phenylethynyl) pyridine (MPEP) is used as an antagonist for mGluR5 and NMDAR [47]. Moreover, MPEP acts as an anxiolytic and antidepressant agent by altering the interactions between mGluR5 and the $\mu$-opioid receptor [48]. It has been reported that MPEP induced LTP in visual cortex fastspiking GABAergic neurons by enhancing intracellular $\mathrm{Ca}^{2+}$ concentration [49]. Thus, we hypothesized that ppLFS-induced LTD in mouse visual cortex after pairing with acetylcholine may depend on mGluR5. Indeed, after bath application of $10 \mu \mathrm{M}$ MPEP onto a ChAT-Cre/Ai32(ChR2-eYFP) homozygous brain slice in the presence of light stimulation, acetylcholine-induced LTD was significantly reduced $(101.3 \pm 6.9 \%$ of baseline; $\mathrm{n}=7 ; * \star * \mathrm{P}<0.001$, unpaired t-test) (Fig. 2c). Comparison of LTD magnitude also showed a significant reduction in acetylcholine-induced LTD in visual cortex (MPEP=101.3 $\pm 6.9 \%(n=7)$; ACh= 48.2 $\pm 9.9 \%(n=6),{ }^{* \star *} P<0.001$, unpaired t-test) (Fig. 2d). These data suggest that ppLFS can induce LTD in 
mice visual cortex in the presence of acetylcholine and that this is mGluR5 dependent, but not NMDAR dependent.

\subsection{NMADR independent serotonin and norepinephrine mediated LTD in mice visual cortex}

Since acetylcholine induced LTD in mouse visual cortex, we hypothesized that serotonin and norepinephrine might also be involved in LTD induction. Tph2-Cre/Ai32(ChR2-eYFP) homozygous brain slices were used for the release of serotonin, while wild-type (C57BL/6) mice were used as a control in serotonergic LTD experiments. Both Tph2-Cre/Ai32(ChR2-eYFP) homozygous and wild-type (C57BL/6) brain slices went through optical stimulation with ppLFS, and neurons were patched from the layer $2 / 3$ visual cortex. After 10 minutes' baseline recoding, low-frequency stimulation (ppLFS, $1 \mathrm{~Hz}, 15 \mathrm{~min}$ ) in combination with $473 \mathrm{~nm}$ blue light stimulation was applied in Tph2-Cre/Ai32(ChR2-eYFP) homozygous

brain slices in the presence of AP5. Upon serotonin release due to optical stimulation, ppLFS induced LTD ( $50.1 \pm 5.2 \%$ of baseline; $n=12 ; * \star * P<0.001$, unpaired t-test) in the visual cortex compared to the base line value $98.7 \pm 3.3(n=10)$ in the Tph2-Cre/Ai32(ChR2-eYFP) brain slices (Fig. 3a). A comparison of LTD magnitude revealed the difference in LTD induction either in the presence or absence of serotonin (Fig. 3b).

In noradrenergic LTD system, the Thi-Cre/Ai32(ChR2-eYFP) homozygous brain slices were used for the release of norepinephrine, while wild-type (C57BL/6) brain slices were used as control. Both ThiCre/Ai32(ChR2-eYFP) homozygous and wild-type (C57BL/6) brain slices went through optical stimulation with ppLFS. After 10 minutes' baseline recording, low-frequency stimulation (ppLFS, $1 \mathrm{~Hz}, 15 \mathrm{~min}$ ) in combination with $473 \mathrm{~nm}$ blue light stimulation was applied in the presence of AP5. In ThiCre/Ai32(ChR2-eYFP) homozygous mice, upon release of norepinephrine due to optical stimulation, ppLFS induced LTD $(62.8 \pm 8.07 \%$ of baseline; $n=14 ; * \star \star P<0.001$, unpaired $t$-test $)$ in the visual cortex compared to the baseline value $109.4 \pm 11.9(n=12)$ (Fig. 3c). A comparison of LTD magnitude revealed the induction of LTD in the presence of norepinephrine, while wild-type (C57BL/6) brain slices failed to show any LTD (Fig. 3d).

Next, we compared the LTD magnitude induced by acetylcholine, serotonin, and norepinephrine. LTD magnitude for acetylcholine $(51.5 \pm 5.7 \%)$ and serotonin $(50.1 \pm 5.2 \%)$ showed no significant difference. However, LTD magnitude between serotonin $(50.1 \pm 5.2 \%)$ and norepinephrine $(62.8 \pm 8.07 \%)$ are statistically different $(P<0.01$; unpaired t-test) (Fig. 3e). These data suggest that acetylcholine and serotonin are more prominent than norepinephrine in inducing LTD in mouse visual cortex.

\subsection{Metal electrode evokes similar amplitude but faster decay time than theta glass electrode}

IPSCs were evoked from dentate gyrus (DG) granule cells via stimulation either by metal or theta glass electrode in hilus in the presence of APV (DL-2-Amino-5-phosphonopentanoic acid, $25 \mu \mathrm{M}$ ) and CNQX (6Cyano-7-nitroquinoxaline-2,3-dione disodium, $10 \mu \mathrm{M}$ ). Single-pulse stimulation is used to evoke IPSC in the hilus. Individual cells evoked by theta glass electrode were displayed in Fig 4a after normalization to baseline. After normalization to baseline different cells showed different values such as 0.7-0.9 (i. e., 70- 
$90 \%$ of baseline). The normalized value for the individual cell was $(n=6)$ averaged to have the final trace (Fig. 4a). Similarly, ten individual cells were evoked via a metal stimulator (a bipolar tungsten electrode) by using the same single-pulse stimulation in the hilus (Fig. 4b). Like theta glass stimulator, after normalization to the baseline, different cells showed different values. The ten normalized values for individual cells were averaged to have the final trace evoked by the metal electrode (Fig. 4b). After merging the averaged traces evoked by the theta glass and metal electrode, we observed the same eIPSC amplitude with different decay times. The averaged trace evoked by theta glass electrode showed slower decay times, while the averaged trace evoked by metal electrode showed faster decay times compared to each other. These data suggest that evoked events generated either by theta glass or metal electrode share the same trace amplitude but not the decay time.

\section{Conclusion}

It is well established that memory formation and information storage require long-term changes in synaptic transmission. Reinforcement learning (RL) helps to avoid punishment and maximize reward. One major problem in $\mathrm{RL}$ is the relationship between stimulus and a delayed reinforcing signal [50]. To link stimulus and its reward across time, synaptic eligibility traces exist, and then specific neuromodulators convert these traces into changes in synaptic efficacy such as LTD, an activitydependent synaptic depression to store information more effectively. Different neuromodulators activate different signaling pathways but can have overlapping interactions even in the same neuron [51]. In some cases, phosphorylation/dephosphorylation of the AMPAR subunit GluA2 is responsible for synaptic depression [52]. Here we showed a novel type of LTD induction in the presence of ACh, SE, and NE in the mice visual cortex which is NADAR independent but mGluR5 dependent. Consistent with our study, another study has shown that during the critical period it is possible to induce LTD in layer IV mammalian visual cortex by using low-frequency stimulation which is independent of inhibitory circuitry [53].

mGluR5 activation in CaMK2a expressing excitatory neurons is responsible for the antidepressant action of Homer1a; enhanced mGlu5 signaling promotes rapid antidepressant effects [47]. As expected, mGluR5-/- mice showed anhedonia and social withdrawal-type depression-like behaviors. Moreover, resilience to chronic stress in nucleus accumbens depends on mGluR5 [54]. Reduction in mGluR5 signaling is associated with increased phosphorylation of serine and tyrosine, which cause desensitization of mGluR5. Hypofunction of mGluR5 is also associated with schizophrenia [55]. In our experiment, in the presence of ACh, SE, or NE, we saw LTD in the visual cortex. It is possible that these neurotransmitters may cause desphophorylation of mGluR5, which is responsible for the induction of LTD in the visual cortex.

In the Fmr1 knockout (KO) mouse, mGluR5 showed enhanced translocation at hippocampal synapses which increased co-clustering with NMDAR at the synaptic surface. This phenomenon is associated with reduced amplitude of synaptic NMDAR currents [56]. In Fmr1 ${ }^{-/ y}$ mice, AMPA receptor internalization and LTD are mediated by mGluR activation without spine shrinkage. On the other hand, NMDAR activation caused LTD together with spine shrinkage in both types of mice [57]. mGluR5 also regulates social 
affiliation and social memory as unmasked by septal deletion of mGluR5, which abolished sociability [58]. Autism spectrum disorders (ASD)-like behavior is also correlated with altered mGluR5 and mGluR5Homer scaffolds mediated signaling in striatal neurons of Shank3-KO mice [59]. These data suggest that mGluR5 and NMDAR follow distinct signaling paths for enhanced cognition, as reflected in this experiment, where ACh induces mGluR5 dependent but not NMDAR independent LTD in mice visual cortex.

In Pavlovian learning, the absence of NMDAR in dopamine neurons did not attenuate conditioned approach [60]. Moreover, refinement of neuronal connections and synaptic remodeling are enmeshed with LTD, which is associated with intracellular $\mathrm{Ca}^{2+}$ levels [61]. By modulating the balance between different intracellular signaling systems, ACh, SE, NE may control neural activity which is NMDAR independent but mGluR5 dependent, a novel form of plasticity in the visual cortex. This may also explain the mechanism of antidepressant effects to neuroprotection.

\section{Declarations}

\section{Acknowledgments}

Author acknowledges the manuscript editing by Renee Cockerham, Program Manager, Office of Postdoctoral Scholars, University of Maryland, Baltimore.

\section{Conflict of interest}

The authors declare no conflict of interest.

\section{References}

1. Reijmers LG, Perkins BL, Matsuo N, et al. (2007) Localization of a stable neural correlate of associative memory. Science 317: 1230-1233.

2. Yiu Adelaide P, Mercaldo V, Yan C, et al. (2014) Neurons Are Recruited to a Memory Trace Based on Relative Neuronal Excitability Immediately before Training. Neuron 83: 722-735.

3. Chubykin Alexander A, Roach Emma B, Bear Mark F, et al. (2013) A Cholinergic Mechanism for Reward Timing within Primary Visual Cortex. Neuron 77: 723-735.

4. Dayan P, Niv Y (2008) Reinforcement learning: the good, the bad and the ugly. Curr Opin Neurobiol 18: 185-196.

5. Calabresi P, Maj R, Pisani A, et al. (1992) Long-term synaptic depression in the striatum: physiological and pharmacological characterization. J Neurosci 12: 4224-4233.

6. Lovinger DM, Tyler EC, Merritt A (1993) Short- and long-term synaptic depression in rat neostriatum. J Neurophysiol 70: 1937-1949.

7. Doya K (2002) Metalearning and neuromodulation. Neural Netw 15: 495-506. 
8. Pennartz CM (1995) The ascending neuromodulatory systems in learning by reinforcement: comparing computational conjectures with experimental findings. Brain Res Brain Res Rev 21: 219245.

9. Hasselmo ME (2006) The role of acetylcholine in learning and memory. Current Opinion in Neurobiology 16: 710-715.

10. Gu Q, Singer W (1993) Effects of intracortical infusion of anticholinergic drugs on neuronal plasticity in kitten striate cortex. Eur J Neurosci 5: 475-485.

11. Conner JM, Culberson A, Packowski C, et al. (2003) Lesions of the Basal forebrain cholinergic system impair task acquisition and abolish cortical plasticity associated with motor skill learning. Neuron 38: 819-829.

12. Froemke RC, Merzenich MM, Schreiner CE (2007) A synaptic memory trace for cortical receptive field plasticity. Nature 450: 425-429.

13. Huerta PT, Lisman JE (1995) Bidirectional synaptic plasticity induced by a single burst during cholinergic theta oscillation in CA1 in vitro. Neuron 15: 1053-1063.

14. Dennis SH, Pasqui F, Colvin EM, et al. (2016) Activation of Muscarinic M1 Acetylcholine Receptors Induces Long-Term Potentiation in the Hippocampus. Cerebral Cortex 26: 414-426.

15. Sugisaki E, Fukushima Y, Fujii S, et al. (2016) The effect of coactivation of muscarinic and nicotinic acetylcholine receptors on LTD in the hippocampal CA1 network. Brain Research 1649: 44-52.

16. Usuda K, Kawase T, Shigeno Y, et al. (2018) Hippocampal metabolism of amino acids by L-amino acid oxidase is involved in fear learning and memory. Scientific Reports 8.

17. Pinto L, Goard MJ, Estandian D, et al. (2013) Fast modulation of visual perception by basal forebrain cholinergic neurons. Nature Neuroscience 16: 1857-1863.

18. Hangya B, Ranade Sachin P, Lorenc M, et al. (2015) Central Cholinergic Neurons Are Rapidly Recruited by Reinforcement Feedback. Cell 162: 1155-1168.

19. Warburton EC, Koder T, Cho K, et al. (2003) Cholinergic Neurotransmission Is Essential for Perirhinal Cortical Plasticity and Recognition Memory. Neuron 38: 987-996.

20. Miyazaki K, Miyazaki KW, Doya K (2011) Activation of Dorsal Raphe Serotonin Neurons Underlies Waiting for Delayed Rewards. Journal of Neuroscience 31: 469-479.

21. Li Y, Zhong W, Wang D, et al. (2016) Serotonin neurons in the dorsal raphe nucleus encode reward signals. Nature Communications 7.

22. Miyazaki K, Miyazaki KW, Yamanaka A, et al. (2018) Reward probability and timing uncertainty alter the effect of dorsal raphe serotonin neurons on patience. Nature Communications 9.

23. Tanaka SC, Shishida K, Schweighofer N, et al. (2009) Serotonin Affects Association of Aversive Outcomes to Past Actions. Journal of Neuroscience 29: 15669-15674.

24. Wei C, Han X, Weng D, et al. (2018) Response dynamics of midbrain dopamine neurons and serotonin neurons to heroin, nicotine, cocaine, and MDMA. Cell Discovery 4. 
25. Samuels BA, Anacker $\mathrm{C}$, Hu A, et al. (2015) 5-HT1A receptors on mature dentate gyrus granule cells are critical for the antidepressant response. Nature Neuroscience 18: 1606-1616.

26. Teixeira CM, Rosen ZB, Suri D, et al. (2018) Hippocampal 5-HT Input Regulates Memory Formation and Schaffer Collateral Excitation. Neuron 98: 992-1004.e1004.

27. Sara Susan J, Bouret S (2012) Orienting and Reorienting: The Locus Coeruleus Mediates Cognition through Arousal. Neuron 76: 130-141.

28. Bouret S, Richmond BJ (2015) Sensitivity of Locus Ceruleus Neurons to Reward Value for GoalDirected Actions. Journal of Neuroscience 35: 4005-4014.

29. Hu H, Real E, Takamiya K, et al. (2007) Emotion Enhances Learning via Norepinephrine Regulation of AMPA-Receptor Trafficking. Cell 131: 160-173.

30. Liu YU, Ying Y, Li Y, et al. (2019) Neuronal network activity controls microglial process surveillance in awake mice via norepinephrine signaling. Nature Neuroscience 22: 1771-1781.

31. Chen C, Jiang Z, Fu X, et al. (2019) Astrocytes Amplify Neuronal Dendritic Volume Transmission Stimulated by Norepinephrine. Cell Reports 29: 4349-4361.e4344.

32. O'Dell TJ, Connor SA, Guglietta R, et al. (2015) $\beta$-Adrenergic receptor signaling and modulation of long-term potentiation in the mammalian hippocampus. Learning \& Memory 22: 461-471.

33. Oliet SHR, Malenka RC, Nicoll RA (1997) Two Distinct Forms of Long-Term Depression Coexist in CA1 Hippocampal Pyramidal Cells. Neuron 18: 969-982.

34. Li Z, Jo J, Jia J-M, et al. (2010) Caspase-3 Activation via Mitochondria Is Required for Long-Term Depression and AMPA Receptor Internalization. Cell 141: 859-871.

35. Clem RL, Huganir RL (2010) Calcium-Permeable AMPA Receptor Dynamics Mediate Fear Memory Erasure. Science 330: 1108-1112.

36. Kim J, Lee S, Park K, et al. (2007) Amygdala depotentiation and fear extinction. Proceedings of the National Academy of Sciences 104: 20955-20960.

37. Han J, Kesner P, Metna-Laurent M, et al. (2012) Acute Cannabinoids Impair Working Memory through Astroglial CB1 Receptor Modulation of Hippocampal LTD. Cell 148: 1039-1050.

38. Gutlerner JL, Penick EC, Snyder EM, et al. (2002) Novel Protein Kinase A-Dependent Long-Term Depression of Excitatory Synapses. Neuron 36: 921-931.

39. Casado M, Isope P, Ascher P (2002) Involvement of Presynaptic N-Methyl-D-Aspartate Receptors in Cerebellar Long-Term Depression. Neuron 33: 123-130.

40. Wang YT, Linden DJ (2000) Expression of Cerebellar Long-Term Depression Requires Postsynaptic Clathrin-Mediated Endocytosis. Neuron 25: 635-647.

41. Tanaka K, Khiroug L, Santamaria F, et al. (2007) Ca2+ Requirements for Cerebellar Long-Term Synaptic Depression: Role for a Postsynaptic Leaky Integrator. Neuron 54: 787-800.

42. Feldman DE, Nicoll RA, Malenka RC, et al. (1998) Long-Term Depression at Thalamocortical Synapses in Developing Rat Somatosensory Cortex. Neuron 21: 347-357. 
43. Bagshaw EV, Evans MH (1976) Measurement of current spread from microelectrodes when stimulating within the nervous system. Experimental Brain Research 25.

44. Haack N, Durry S, Kafitz KW, et al. (2015) Double-barreled and Concentric Microelectrodes for Measurement of Extracellular Ion Signals in Brain Tissue. Journal of Visualized Experiments.

45. Kirkwood A, Bear MF (1994) Homosynaptic long-term depression in the visual cortex. J Neurosci 14: 3404-3412.

46. Karmarkar UR, Dan Y (2006) Experience-Dependent Plasticity in Adult Visual Cortex. Neuron 52: 577585.

47. Holz A, Mülsch F, Schwarz MK, et al. (2019) Enhanced mGlu5 Signaling in Excitatory Neurons Promotes Rapid Antidepressant Effects via AMPA Receptor Activation. Neuron 104: 338-352.e337.

48. Pilc A, Klodzinska A, Branski P, et al. (2002) Multiple MPEP administrations evoke anxiolytic- and antidepressant-like effects in rats. Neuropharmacology 43: 181-187.

49. Sarihi A, Mirnajafi-Zadeh J, Jiang B, et al. (2012) Cell Type-Specific, Presynaptic LTP of Inhibitory Synapses on Fast-Spiking GABAergic Neurons in the Mouse Visual Cortex. Journal of Neuroscience 32: 13189-13199.

50. Fisher SD, Robertson PB, Black MJ, et al. (2017) Reinforcement determines the timing dependence of corticostriatal synaptic plasticity in vivo. Nature Communications 8.

51. Li X, Bucher D, Nadim F (2018) Distinct Co-Modulation Rules of Synapses and Voltage-Gated Currents Coordinate Interactions of Multiple Neuromodulators. The Journal of Neuroscience 38: 8549-8562.

52. Chung HJ (2003) Requirement of AMPA Receptor GluR2 Phosphorylation for Cerebellar Long-Term Depression. Science 300: 1751-1755.

53. Dudek SM, Friedlander MJ (1996) Developmental Down-Regulation of LTD in Cortical Layer IV and Its Independence of Modulation by Inhibition. Neuron 16: 1097-1106.

54. Shin S, Kwon O, Kang JI, et al. (2015) mGluR5 in the nucleus accumbens is critical for promoting resilience to chronic stress. Nature Neuroscience 18: 1017-1024.

55. Wang H-Y, MacDonald ML, Borgmann-Winter KE, et al. (2018) mGluR5 hypofunction is integral to glutamatergic dysregulation in schizophrenia. Molecular Psychiatry 25: 750-760.

56. Aloisi E, Le Corf K, Dupuis J, et al. (2017) Altered surface mGluR5 dynamics provoke synaptic NMDAR dysfunction and cognitive defects in Fmr1 knockout mice. Nature Communications 8.

57. Thomazeau A, Bosch M, Essayan-Perez S, et al. (2020) Dissociation of functional and structural plasticity of dendritic spines during NMDAR and mGluR-dependent long-term synaptic depression in wild-type and fragile $\mathrm{X}$ model mice. Molecular Psychiatry.

58. Mesic I, Guzman YF, Guedea AL, et al. (2015) Double Dissociation of the Roles of Metabotropic Glutamate Receptor 5 and Oxytocin Receptor in Discrete Social Behaviors. Neuropsychopharmacology 40: 2337-2346. 
59. Wang X, Bey AL, Katz BM, et al. (2016) Altered mGluR5-Homer scaffolds and corticostriatal connectivity in a Shank3 complete knockout model of autism. Nature Communications 7.

60. Parker JG, Zweifel LS, Clark JJ, et al. (2010) Absence of NMDA receptors in dopamine neurons attenuates dopamine release but not conditioned approach during Pavlovian conditioning. Proceedings of the National Academy of Sciences 107: 13491-13496.

61. Mulkey RM, Endo S, Shenolikar S, et al. (1994) Involvement of a calcineurin/ inhibitor-1 phosphatase cascade in hippocampal long-term depression. Nature 369: 486-488.

\section{Figures}


a

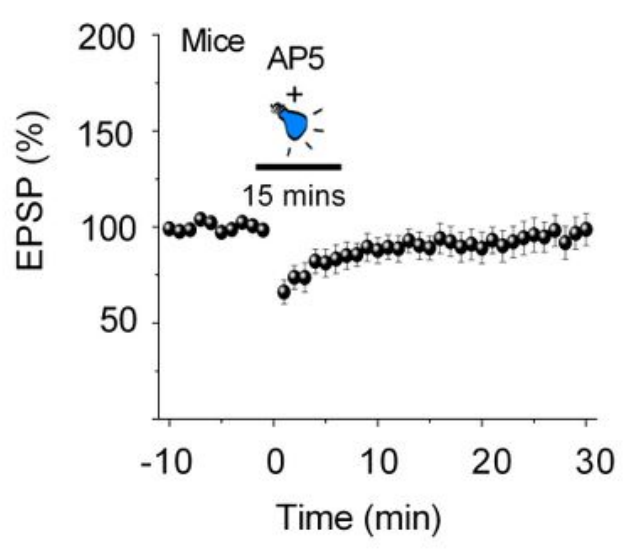

C

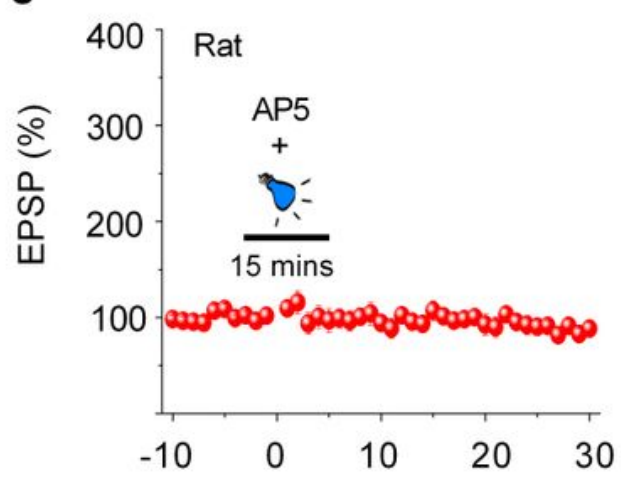

b

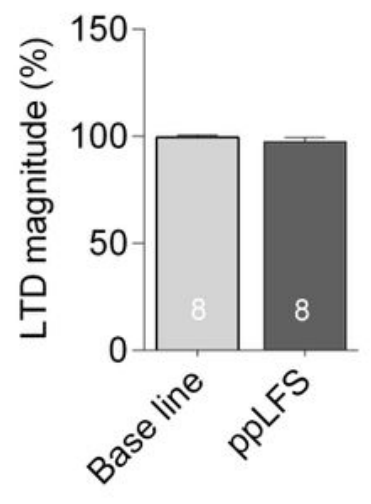

d

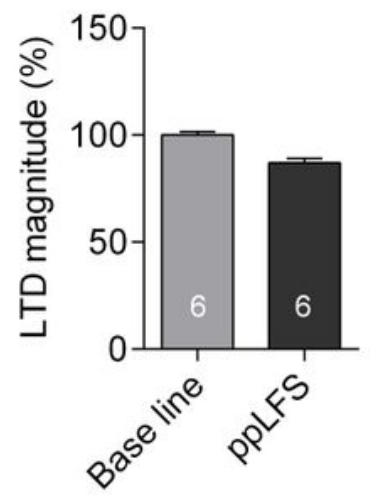

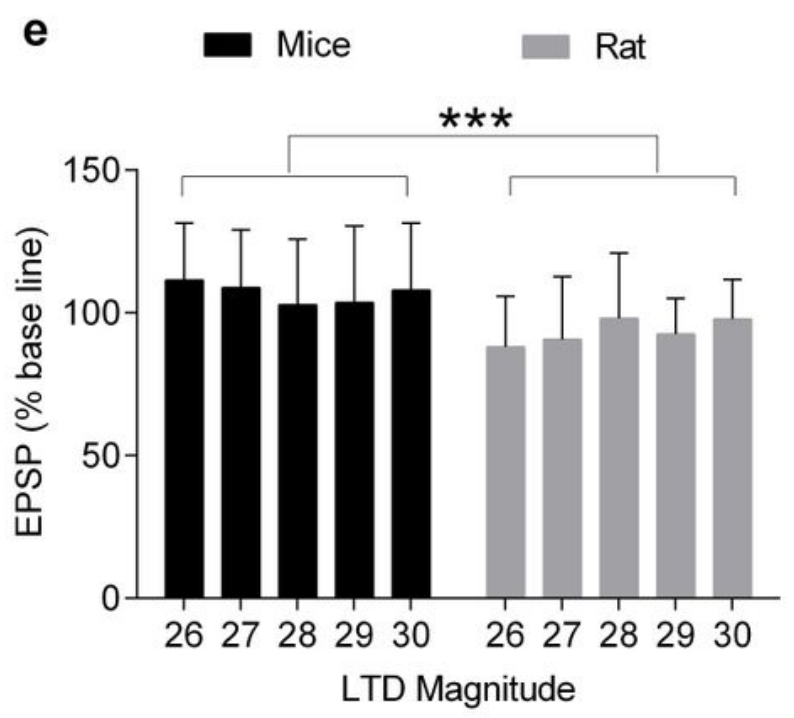

\section{Figure 1}

Time-courses of the whole-cell evoked excitatory postsynaptic potential (eEPSPs) in layer $2 / 3$ of the visual cortex from P22-40 wild type (C57BL/6) mice are shown with sample traces obtained during the baseline period of 10 min after paired-pulse LFS in combination with optogenetic stimulation. Records were taken every $10 \mathrm{~s}$, and $1 \mathrm{~min}$ intervals were averaged for amplitude evaluation. The labeled solid bar with a bulb indicates a period of 15 minutes of low-frequency stimulation with $473 \mathrm{~nm}$ light stimulation. 
(a) ppLFS pairing with $473 \mathrm{~nm}$ light stimulation in wild type (C57BL/6) mice fails to induce any LTD in the visual cortex in the presence of AP5. (b) Comparison of LTD magnitudes with baseline in mouse visual cortex (Base $=100 \%$; ppLFS $=97.5 \pm 1.9 \%, n=10)$. (c) ppLFS pairing with $473 \mathrm{~nm}$ light stimulation in wild type (C57BL/6) rat fails to induce any LTD in the visual cortex. (d) Comparison of LTD magnitudes with baseline in rat (Base $=100 \%$; ppLFS= $93.4 \pm 1.9 \%, n=6)$. (e) Comparison of LTD magnitudes between mouse and rat (mice $=97.5 \pm 1.9 \%$; rat $=93.4 \pm 1.9 \%$ ). Two-tailed t-test. ${ }^{\star} \star \star P ख 0.001 ;$ Data are mean \pm s.e.m.

a

b
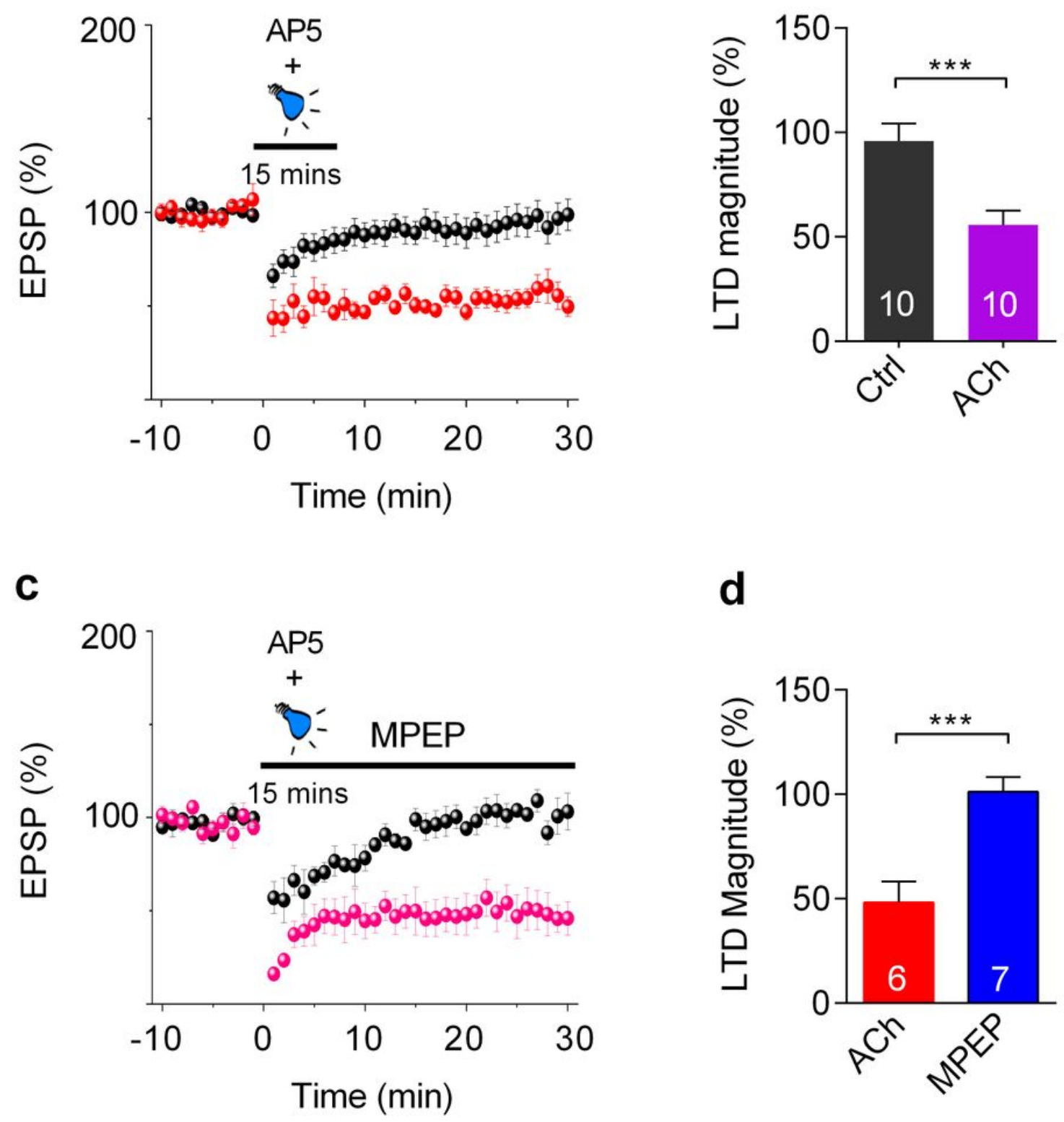

Figure 2

mGluR5 dependent acetylcholine (ACh) mediated transformation of evoked EPSP traces to LTD. (a) In the visual cortex, ppLFS conditioning alone did not affect synaptic strength, while conditioning with $473 \mathrm{~nm}$ 
light stimulation in the brain slice from ChAT-Cre/Ai32(ChR2-eYFP) mice induced LTD in the presence of AP5. (b) Comparison of LTD magnitudes in visual cortex between wild type (C57BL/6) and ChAT-

Cre/Ai32(ChR2-eYFP) mice (Ctrl: $96.6 \pm 5.5 \%$; ACh: $51.5 \pm 5.7 \%$ of baseline; $n=10)$. (c) In the presence of MPEP, ppLFS conditioning with $473 \mathrm{~nm}$ light stimulation in the brain slice from ChAT-Cre/Ai32(ChR2eYFP) mice failed to induce LTD. (d), Comparison of LTD magnitudes in visual cortex from ChATCre/Ai32(ChR2-eYFP) mice in the presence or absence of MPEP [(ACh= 48.2 $\pm 9.9 \%(n=6)$; MPEP=101.3 \pm $6.9 \%(n=7)]$; Two-tailed t-test. ***P $₫ 0.001$; Data are mean \pm s.e.m.

a
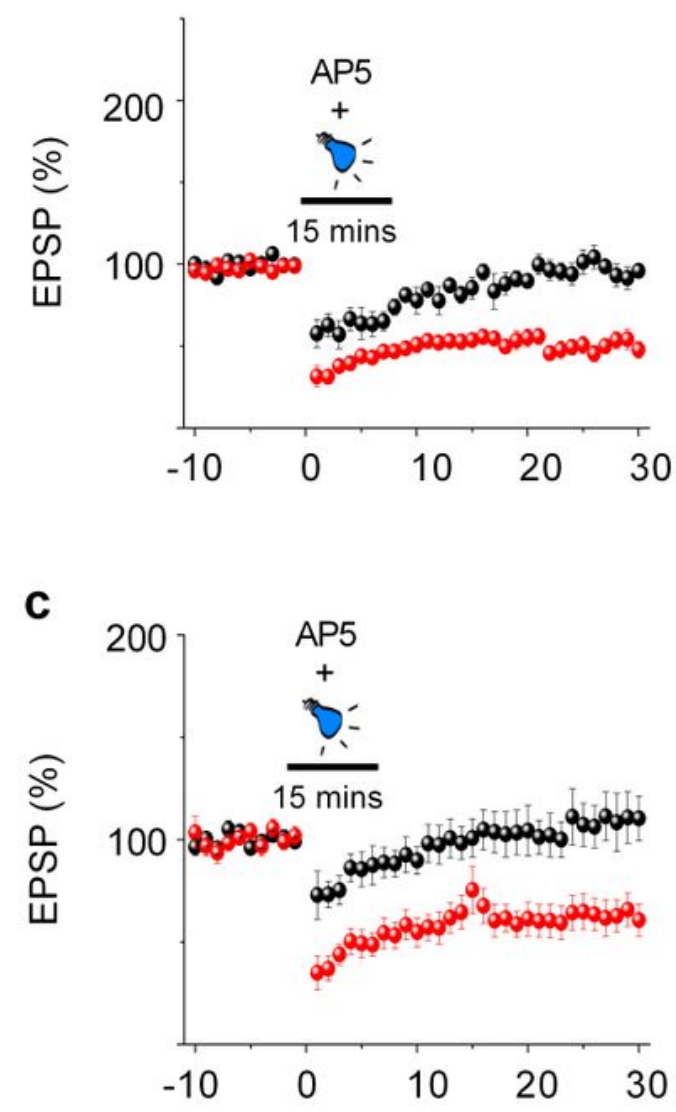

b

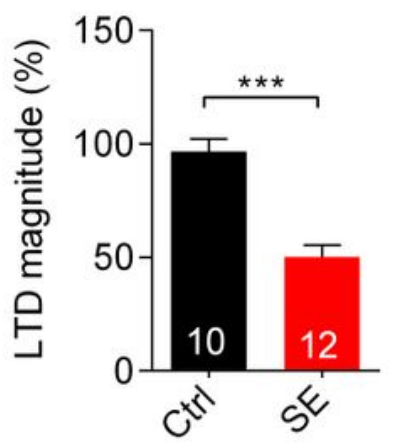

d

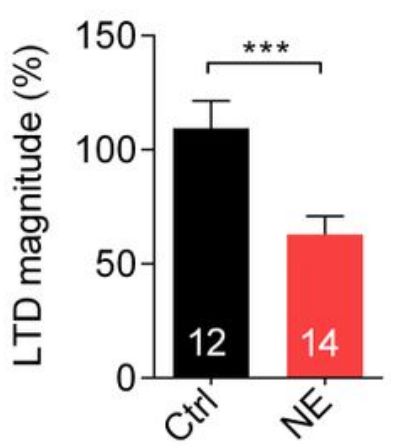

e

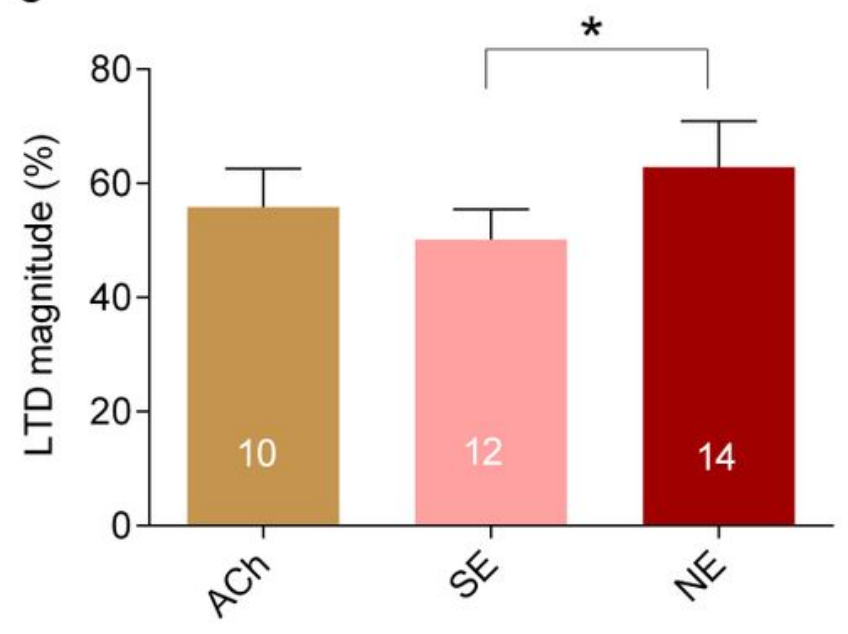




\section{Figure 3}

Serotonin (SE) and norepinephrine (NE) transform evoked EPSP traces to LTD in the presence of AP5. (a) In the visual cortex, ppLFS conditioning alone did not affect synaptic strength, while conditioning with $473 \mathrm{mn}$ light stimulation in the brain slice from Tph2-Cre/Ai32(ChR2-eYFP) mice induced LTD in the presence of AP5. (b) Comparison of LTD magnitudes in visual cortex between wild type (C57BL/6) and Tph2-Cre/Ai32(ChR2-eYFP) mice [(Ctrl: $98.7 \pm 3.3$ ( $n=10)$; SE: $50.1 \pm 5.2 \%$ of baseline, $(n=12)$ ]. (c) In the visual cortex, ppLFS conditioning alone did not affect synaptic strength, while conditioning with $473 \mathrm{mn}$ light stimulation in the brain slice from Thi-Cre/Ai32(ChR2-eYFP) mice induced LTD in the presence of AP5. (d) Comparison of LTD magnitudes in visual cortex between wild type (C57BL/6) and ThiCre/Ai32(ChR2-eYFP) [(Ctrl: $109.4 \pm 11.9$ ( $n=12)$; NE: $62.8 \pm 8.07 \%$ of baseline, $(n=14)$ ]. (e) Comparison of LTD magnitudes in the visual cortex among ChAT-Cre/Ai32(ChR2-eYFP); Tph2-Cre/Ai32(ChR2-eYFP); ThiCre/Ai32(ChR2-eYFP) mice. Two-tailed t-test. *Pख0.05, ***Pख0.001; Data are mean \pm s.e.m. 


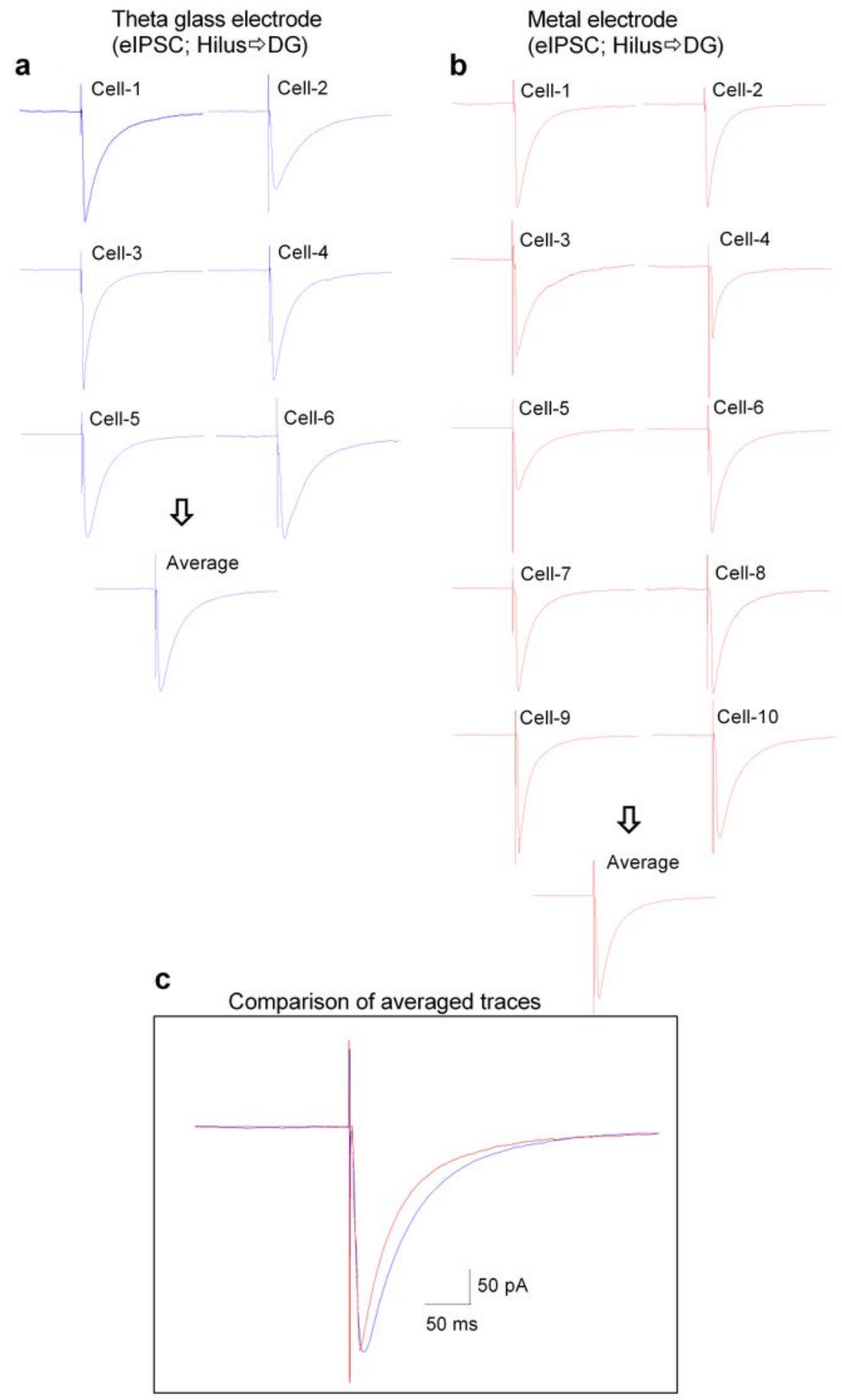

\section{Figure 4}

Theta glass and metal electrodes evoked the same eIPSC amplitude with different decay times in the hippocampus. (a) IPSCs were evoked in DG granule cells by placing the theta glass electrode into the hilus. Six individual traces are displayed from 6 cells, while the average trace is shown at the bottom. (b) IPSCs were evoked in DG granule cells by placing the concentric bipolar tungsten electrode into the hilus. 
Ten individual traces are displayed from 10 cells, while the average trace is shown at the bottom. (c) Comparison of the averaged IPSC traces evoked by Theta glass and metal electrodes. 